\title{
PREOPERATIVE DETERMINATION OF TIBIA LENGTH USING CONTRALATERAL TIBIAL TUBERCLE-MEDIAL MALLEOLAR DISTANCE [TMID] IN WESTERN PART OF NEPAL
}

Kishor Man Shrestha, ${ }^{1}$ Prakriti Raj Kandel, ${ }^{1}$ Bipan Shrestha, ${ }^{1}$ Shreshal Shrestha ${ }^{1}$

\section{ABSTRACT}

\section{INTRODUCTION}

Intramedullary (IM) nailing is most commonly preferred for adult tibial shaft fracture. The objective of this study was to find the accuracy of predicting the tibial nail length pre-operatively from contra-lateral tibial tubeorsity to medial malleolar distance (TMD) length.

\section{MATERIAL AND METHODS}

After ethical clearance (UCMS/IRC/239/19) from the Institutional Review Board (IRB) of Universal College of Medical Sciences, all patients with recent fracture of tibia, who fulfilled the inclusion criteria were enrolled in the study and the informed written consent was obtained. The study was conducted on 45 patients of aged more than 15 years who attended Universal College of Medical Sciences Teaching Hospital (UCMS-TH) over a period of one year (Nov 2019 to Oct 2020 ). The TMD length of contra-lateral leg were measured preoperatively using a measuring tape and the ideal length of IM nail were measured intra-operatively using IM tibial nail under fluoroscopic guidance.

\section{RESULTS}

Analysis showed that majority of patients were males (77.78\%) out of total 45 patients. The mean length of TMD length of contra-lateral leg and the ideal length of IM nail were $33.067 \pm 1.60$ and $33.11 \pm 1.68$ respectively. The mean difference between these two measurements was 0.04 (95\% CI, -0.64 to 0.72$)$. The significant correlation was found between these two measurements i.e $\mathrm{r}=0.88$ by Pearson's correlation ( $95 \% \mathrm{CI}, 0.79$ to 0.93$),(p<0.001)$. Most of the TMD length were equal (37.78\%) to ideal length of IM nail.

\section{CONCLUSION}

We recommended TMD length measurement of contralateral leg as an excellent anthropometric parameter for preoperative planning.

\section{KEYWORDS}

Fracture, Intramedullary nail, Malleolar, Tibia

1. Department of Orthopaedics and Trauma Surgery, Universal College of Medical Sciences, Bhairahawa, Nepal

DOI: http//doi.org/10.3126/jucms.v9i01.37857

For Correspondence

Dr. Kishor Man Shrestha,

Department of Orthopaedics and Trauma Surgery,

Universal College of Medical Sciences

Bhairahawa, Nepal

Email:kishor233@yahoo.com 


\section{INTRODUCTION}

Tibial shaft fractures are the most common long bone fractures in adults comprising $2 \%$ of all fractures in adult. ${ }^{1}$ These fractures are common in males (3:1) with the average age of 37 years. ${ }^{23}$ High energy trauma as road traffic accidents, fall from the height etc and indirect trauma as in sports are the common mechanisms of the injury especially in the younger age group. ${ }^{3}$

Treatment options ranges from the conservative to surgical management. The selection of the treatment depends on the type of the fracture, the energy of the trauma, open fractures, the surgeon's preference and the patient's previous functional status and the treatment preferences. The surgical management includes intramedullary (IM) nailing, external fixation or plating. ${ }^{3}$ As IM nailing has load sharing properties and is the minimal invasive procedure where it escapes the opening at the fracture site, it is the gold standard and commonly used in the shaft fractures. ${ }^{4}$

There are various methods available in the literature for the estimation of the accurate nail length. The two guide wires technique, using a radiographic ruler and nail against limb techniques are the intra-operative methods. ${ }^{5-7}$ Whereas Keammer splint technique, templating, scanograms, spotograms and direct measurement from radiographs of the contra-lateral limb are the preoperative radiological methods that are described. ${ }^{7,8}$ The anthropometric measurements described for the pre-operative estimation of tibial nail length are knee joint line to ankle joint line (K-A), knee joint line to medial malleolus (K-MM), tibial tuberosity to ankle joint line (TT-A), tibial tuberosity to medial malleolus (TMD), olecranon to fifth metacarpal head (O-MH) and body height $(\mathrm{BH})^{8-12}$

The intra-operative methods increase the operative time and add the exposure to the radiation to both the patient and the operative room personnel. Accurate determination of tibial nail length pre-operatively, helps to avoid these problems. Preoperative methods that rely on conventional radiographs can cause inaccuracies due to variation in magnification, position of limb and inadequate exposure. The anthropometric measurement methods provides an easy way preoperatively to estimate the tibial length accurately. ${ }^{10}$

The objective of this study is to find the accuracy of predicting the tibial length pre-operatively from contra-lateral TMD length which ultimately guides to prepare the proper size of IM nail pre-operatively and helps in shortening the operative time and decrease in radiation exposure to both the patient and the operative room personnel.

\section{MATERIAL AND METHODS}

The study was conducted from November 2019 to October 2020 (a period of one year) at Universal College of Medical Sciences Teaching Hospital (UCMS-TH), Bhairahawa,
Nepal. UCMS-TH is a tertiary care hospital situated in the West Nepal near the Nepal- India border. It is a hospital based observational study.

After ethical clearance (UCMS/IRC/239/19) from the Institutional Review Board (IRB) of UCMS-TH, all patients with recent fracture of tibia, who fulfilled the inclusion criteria were enrolled in the study and the informed written consent was obtained. Data collection was done by the researcher.

\section{Sample size and sampling technique}

The sample size was calculated by using Cochran's formula $\mathrm{n}=\mathrm{z}^{2} \mathrm{pq} / \mathrm{d}^{2}$

Where, $\mathrm{z}($ level of significance at $5 \%)=1.96$

$\mathrm{p}($ Incidence of adult tibia fracture $)=2 \%$

$\mathrm{q}=98 \%$

$\mathrm{d}$ (allowable error $)=4.5 \%$

After calculating we get approximately 37 and during our study period 45 cases were found so total sample of 45 cases were taken in our study by using purposive sampling.

\section{Inclusion criteria}

- $\quad$ Age more than 15 years with closure of growth plate

- Fracture shaft of tibia

\section{Exclusion criteria}

- Age less than 15 years with presence of growth plate where IM nailing could not be done

- Bilateral fracture of tibia

- Fracture of tibia involving metaphyseal area where IM nail could not be done

\section{Anthropometric measurements}

Measurement of the contra-lateral TMD was determined by measuring the length between the most prominent points on the medial malleolus and on the tibial tuberosity with the help of a measuring tape.

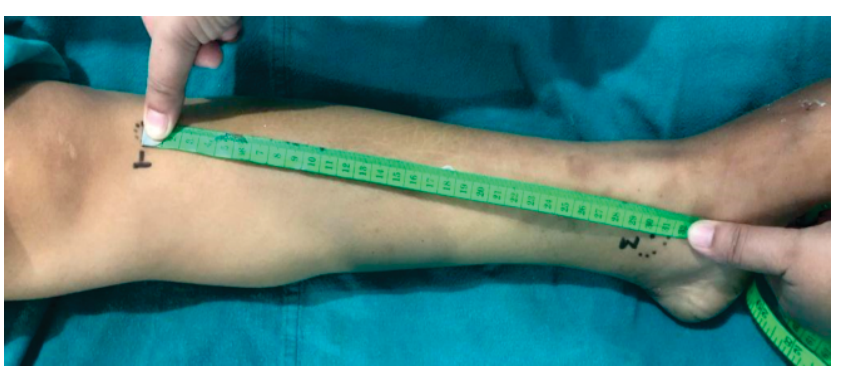

Figure 1. Preoperative measurement of TMD of contralateral leg using measuring tape

\section{Intra-operative measurements}

Before the IM canal of tibia was opened, an estimation of nail length was made by the operating surgeon, using IM tibial nail. The distal tip of nail was held at the level of the physeal scar and the nail length was estimated directly from the fluoroscopic image by selecting the measurement at or just 
below the level of the anterior edge of the tibial plateau., Then the IM canal of each tibia was opened and was reamed as appropriate after the reduction of the fracture. The appropriate size IM nail of tibia was inserted under the fluoroscopic guidance.

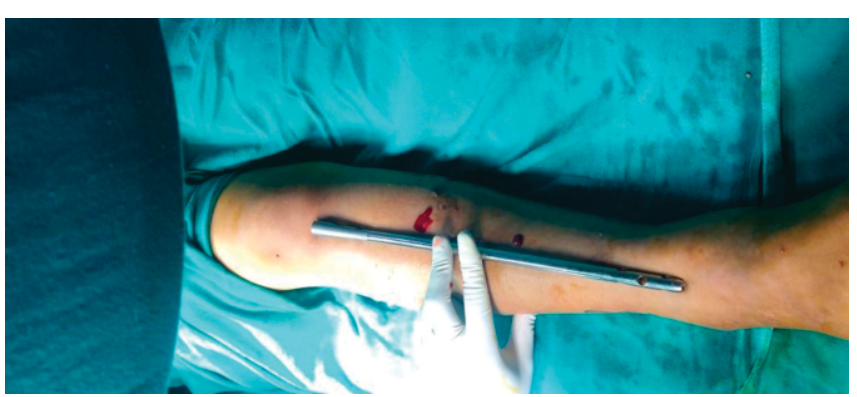

Figure 2. Intra-operative estimation of tibial nail length using IM nail under fluoroscopic guidance

\section{Ideal nail length}

A literature review suggests that the ideal-length implanted tibial nail would rest $5-10 \mathrm{~mm}$ below the proximal tibial articular surface and 10-20 $\mathrm{mm}$ above the distal tibial articular surface. For this study, we considered the ideal nail as being 10 $\mathrm{mm}$ from the proximal tibial articular surface and $10 \mathrm{~mm}$ from the distal tibial articular surface. This would give us an adequate working length but would also avoid the associated complications of excess nail length. Specially designed proforma was filled for each patient. Statistical analysis was done by using Statistical Package for the Social Sciences Software (SPSS) Program for windows version 20. The results were presented in tables and charts using the Microsoft Excel 2010 software.

\section{RESULTS}

Total 45 patients with age more than 15 years attended UCMS$\mathrm{TH}$ with the fracture of shaft of tibia over one-year period (November 2019 to October 2020). Out of these $77.78 \%$ (35) were males and $22.22 \%$ (10) were females.

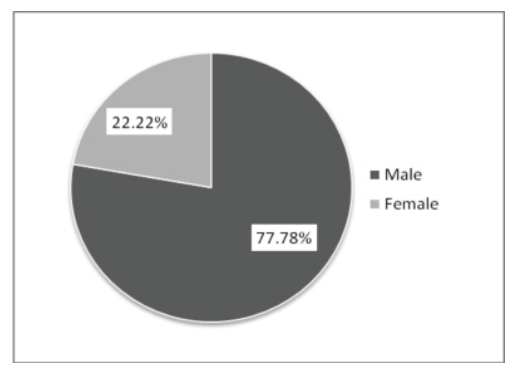

\section{Figure 3. Gender distribution}

The mean TMD of contra-lateral leg was $33.07 \pm 1.60$ and the mean ideal length of IM nail was $33.11 \pm 1.68$. The mean difference between these two measurements was $0.04(95 \%$ CI, -0.64 to 0.72 ).
Table 1. Correlation between the ideal length of IM nail and TMD length of contralateral leg

\begin{tabular}{|c|c|c|c|c|}
\hline Length & Mean $\pm S D$ & Mean difference & Correlation coefficient & t $p$-value \\
\hline $\begin{array}{l}\text { Ideal length of } \\
\text { IM nail }\end{array}$ & $33.11 \pm 1.68$ & 0.04 & 0.88 & $p<0.001$ \\
\hline TMD & $33.067 \pm 1.60$ & ( $95 \%$ CI, -0.64 to & $(95 \%$ CI, 0.79 to 0.93$)$ & \\
\hline
\end{tabular}

There was highly significant strong positive correlation between these two measurements i.e. $\mathrm{r}=0.88$ by Pearson's correlation ( $95 \% \mathrm{CI}, 0.79$ to 0.93$), p<0.001$

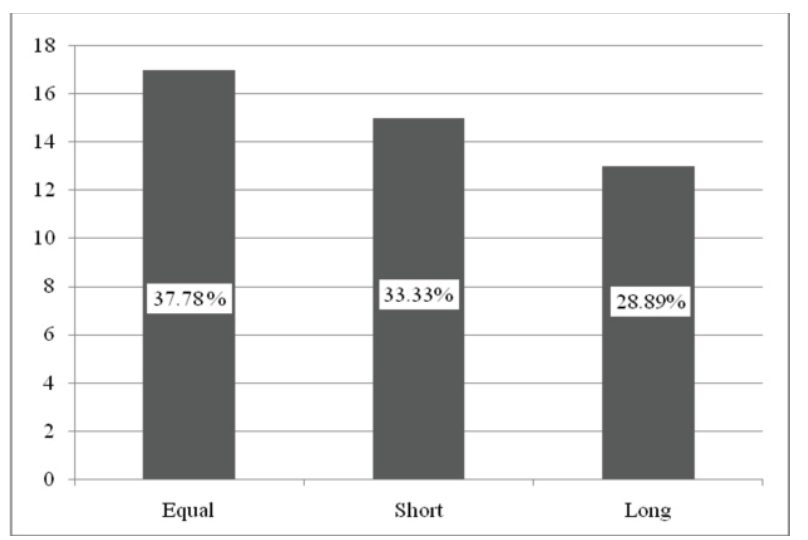

Figure 4. Comparison of measured TMD length of contralateral leg with the ideal tibial nail length

The measured TMD length of contralateral leg was equal to the ideal tibial length in $37.78 \%$ (17) of patients whereas shorter and longer than the ideal tibial length were in $33.33 \%$ (15) and $28.89 \%$ (13) of patients respectively.

\section{DISCUSSION}

There are several anthropometric methods that are described for the preoperative estimation of the length of the tibial nail. One of the simple and accurate method is direct or indirect measurement of the distance between the TMD of the contralateral leg., ${ }^{8,11,14}$ The aim of our study was to compare the measurement with the TMD length of contra-lateral leg for preoperative determination of tibial nail length.

Due to the magnification on conventional radiographs techniques, there are chances of error in the estimation of nail length. ${ }^{13}$ According to Galbraith et al intra-operative techniques as the guide wire methods and the radiographic ruler have an excellent accuracy. But there is a added problem of added exposure to radiation to operative room personnel as well as increased operative time. ${ }^{9,11}$

Various studies have been carried out on anthropometric measurements methods for detecting the accuracy of preoperative assessment of tibial length. ${ }^{8-10}$ Among the four methods tested, including full length scanograms, spotograms, acrylic template overlays and TMD length, Colen 
and Prieskorn ${ }^{8}$ found the TMD length to be the most accurate (71\%). Whereas $64 \%$ and $38 \%$ accuracy was reported by Venkateswaran et $\mathrm{al}^{10}$ and Galbraith et $\mathrm{al}^{11}$ respectively.

In our study, we found the accuracy of TMD was $37.78 \%$. Comparing with the measured TMD length one size below and one size above the ideal length was $33.33 \%$ and $28.89 \%$ respectively. More over preparing the tibial IM nail of sizes one size below and one size above the measured TMD in addition will be beneficial before the surgery.

\section{CONCLUSION}

We found a significant correlation between the anthropometric parameters, TMD length measured and the ideal nail length. So preparing the tibia nail one size below and above of the measured TMD length of contra-lateral leg, this method is an easy, fast and accurate method of preoperative planning before IM tibia nailing.

\section{LIMITATIONS}

We have taken only one anthropometric parameter to find out the tibial nail length preoperatively. However, there are other anthropometric parameters also mentioned in the literature. In addition to that the sample size is small to conclude the result for the whole population of the Nepal. Thus further research with more sample sizes in various parts of Nepal with comparison with all other parameters is required.

\section{CONFLICT OF INTEREST}

The authors declare no conflict of interest in publication of this paper.

\section{REFRENCES}

1. Court-Brown CM, Caesar B. Epidemiology of adult fractures: a review. Injury. 2006;37:691-7.

2. Court-Brown CM, McBirnie J. The epidemiology of tibial fractures. J Bone Joint Surg Br. 1995;77(3):417-21.

3. Court-Brown CM. Fractures of the Tibia and fibula. In: Bucholz RW, Heckman JD, Court-Brown CM, et al, eds. Rockwood and Green's Fractures in Adults, 6th ed. Philadelphia, PA: Lippincott Williams and Wilkins. 2010:2017-2318.

4. Hernandez-Vaquero D, Suarez-Vazquez A, Iglesias-Fernandez $\mathrm{S}$, et al. Dynamisation and early weight- bearing in tibial reamed intramedullary nailing: its safety and effect on fracture union. Injury. 2012;43:63-7.

5. Krettek C, Schandelmaier P, Rudolf J, et al. Current status of surgical technique for unreamed nailing of tibial shaft fractures with the UTN (unreamed tibia nail). Unfallchirurg. 1994;97:575-599.

6. Waldron VD. Predicting intramedullary nail length. Am J Orthop. 1998;27(5):383.
7. Mosheiff R, Peyser A, Friedman A, et al. "Krammer splint technique" for immediate measuring of intramedullary nails. Am J Orthop (Belle Mead NJ). 1997;26:375.

8. Colen RP, Prieskorn DW. Tibial tubercle-medial malleolar distance in determining tibial nail length. J Orthop Trauma. 2000; $14: 345-8$.

9. Galbraith JG, O'Leary DP, Dailey HL, et al. Preoperative estimation of tibial nail length - because size does matter. Injury. 2012;43:1962-8.

10. Venkateswaran B, Warner RM, Hunt N, et al. An easy and accurate preoperative method for determining tibial nail lengths. Injury. 2003;34:752-5.

11. Blair S. Estimating tibial nail length using forearm referencing. Injury. 2005;36:160-2.

12. Fischmeister MF, Lang $\mathrm{T}$, Reichl $\mathrm{C}$, et al. How to predict requisite nail length in tibial fractures. Arch Orthop Trauma Surg. 1994;113:194-5.

13. Krettek C, Blauth M, Miclau T, et al. Accuracy of intramedullary templates in femoral and tibial radiographs. $\mathrm{J}$ Bone Jt Surg Br. 1996;78:963-964.

14. Rudloff MI. Fractures of the lower extremity. In: Canale ST, Beaty JH, eds. Campbell's Operative Orthopaedics. 12th ed. Philadelphia: Elsevier 2013:2654. 